\section{Use of the Denver pleuroperitoneal shunt in the treatment of chylothorax secondary to filariasis}

\author{
N D Kitchen, D B Hocken, \\ R M Greenhalgh, D K Kaplan
}

\begin{abstract}
A 57 year old woman developed a chylothorax as a result of having had filariasis as a child. This failed to respond to conservative measures but was successfully managed by means of a Denver pleuroperitoneal shunt.
\end{abstract}

Chylothorax is uncommon, and filariasis is a rare cause. The case we report here is, we believe, the first to be treated with the pleuroperitoneal shunt.

\section{Case report}

A 57 year old white woman presented with a three month history of progressive shortness of breath on exertion, wheeze, and a dry cough. She had never smoked.

At the age of 5 she had had a self limiting febrile illness while living in Singapore. She was well until the age of 38, when she developed chyluria. A lymphogram at that time showed lymphatic fistulas connecting the left renal pelvis with a surrounding plexus of ectatic lymphatic channels, consistent with

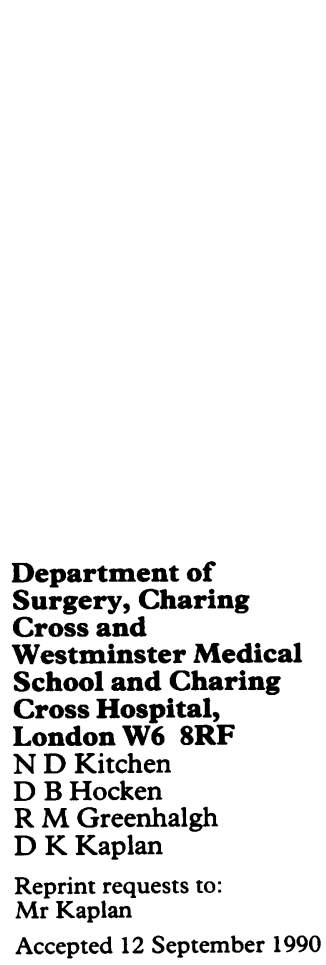

Lymphogram showing features of acquired lymphatic obstruction due to filariasis. filariasis. ${ }^{1}$ At operation these were oversewn and the patient had no further chyluria. She had then remained well until the age of 57.

Clinical examination on presentation revealed signs consistent with a large left pleural effusion. She was not anaemic or febrile, nor was there any palpable lymphadenopathy. The eosinophil count and filarial complement fixation count were normal (a finding that does not exclude previous filarial infection). ${ }^{2}$ Chest radiography and computed tomography confirmed a large left sided pleural effusion but were otherwise non-diagnostic. Thoracocentesis produced $2000 \mathrm{ml}$ of chyle. Cytological examination showed numerous lymphocytes but no malignant cells. Lymphography showed multiple fluid levels due to the filling of cavities within dilated lymphatic ducts in the upper retroperitoneum and posterior mediastinum with the oily contrast medium (Ultra Fluid Lipiodol).

The chylothorax was initially managed conservatively with continuous intercostal tube drainage and total parenteral nutrition. After three weeks on this regimen the effusion resolved; but over the next five months a total of 12 litres of chyle was aspirated despite tetracycline pleurodesis on three occasions.

In view of the failure of conservative management a Denver pleuroperitoneal shunt was inserted under general anaesthesia, after aspiration of a further 2 litres of chyle. The patient was taught to compress the pump chamber, and discharged home on the fifth postoperative day. Seventeen months later she remains symptom free and has required no further therapeutic intervention.

\section{Discussion}

The diagnosis of filarial chylothorax was made in the light of the history of the childhood illness in a tropical area endemic for filariasis. The lymphographic appearances were those of an acquired lymphatic block, with total occlusion of the thoracic duct and "pathognomic" lymphatic lakes at or near the level of the diaphragm. The absence of clinical lymphatic obstruction (lymphoedema) in the legs and torso does not exclude the diagnosis of filariasis. ${ }^{2}$

Patients with chylothorax fall into two groups-those with a normal thoracic duct who have chylothorax secondary to chylous ascites and those with a diseased thoracic duct. ${ }^{23}$ Chylothorax occurs rarely in filariasis and is poorly documented as a feature of the disease (it is not mentioned at all in Manson's classic textbook of tropical diseases). ${ }^{4}$ The visceral leakage of lymph from blocked deep lymphatics occurs far less frequently than superficial lymphoedema and is a late feature usually affecting the urinary tract or peritoneum. $^{5}$ The pathological tests for filariasis rarely give positive results. ${ }^{2}$

The treatment of chylothorax is initially conservative. Most authors recommend a period of no more than two to three weeks' tube drainage and total parenteral nutrition. If the chylothorax fails to resolve within this 
time it is highly unlikely to do so and depletion of lymph leads to progressive inanition of the patient. In this case several months elapsed in this manner because the patient was reluctant to undergo operative treatment.

Traditionally surgery for chylothorax means either radical pleurodesis or identification of the site of leakage by lymphography or radioisotope scanning, followed by direct repair, ligation, or lymphoazygos shunting of the lymphatic fistula at thoracotomy. ${ }^{2}$ In this case a high pressure lymphatic system was thought to be present at the level of the diaphragm, which had previously resulted in chyluria and now presented with chylothorax. The aim of treatment was therefore to decompress rather than to seal the fistula. The pleuroperitoneal shunt enables lymph to be shunted to the greater absorptive surface area of the peritoneal cavity and may thus obviate the need for major surgery without compromising the operative field should thoracotomy subsequently prove necessary.
Pleuroperitoneal shunting has been used in the treatment of recalcitrant chylothorax for almost a decade and its successful use has been described in both congenital neonatal chylothorax $^{6}$ and adult cases ${ }^{7}$ of traumatic and malignant aetiology. We believe, however, that this is the first case in which the use of the pleuroperitoneal shunt has been described in chylothorax secondary to filariasis.

1 Lloyd-Davies RW, Edwards JM, Kinmonth JB. Chyluria Br J Urol 1967;39:566-71.

2 Kinmouth JB. Surgery, lymphography and diseases of the chyle and lymph systems. 2nd ed. London: Arnold, 1981.

3 Robinson CLN. The management of chylothorax. Ann Thorac Surg 1985;39:90-5.

4 Manson-Bahr PEC, Bell RD, eds. Manson's tropical diseases. 19th ed. London: Ballière Tindall, 1987.

5 Maegraith B, ed. Adams and Maegraith: Clinical tropical diseases. 9th ed. Oxford: Blackwell, 1989.

6 Azizkhan RG, Canfield J, Alford BA, Rodgers BM Pleuroperitoneal shunts in the management of neonatal chylothorax. J Pediatr Surg 1983;18:842-50.

7 Milsom JW, Kron IL, Rheuban KS. Chylothorax: an assessment of current surgical practice. $J$ Thorac Cardiovasc Surg 1985;89:221-7.

\section{Hypertrophic pulmonary osteoarthropathy in a patient with pulmonary alveolar microlithiasis}

\author{
Salih Emri, Lütfi Cöplü, Z Toros Selçuk, \\ A Altay Sahin, Y Izzettin Baris
}

\begin{abstract}
Hypertrophic pulmonary osteoarthropathy occurred in a patient with proved pulmonary alveolar microlithiasis, an association not previously reported.
\end{abstract}

Pulmonary alveolar microlithiasis is an uncommon disorder of unknown aetiology, characterised by microliths within the alveoli. Diagnosis is usually made from the radiographic appearance. ${ }^{12}$

Finger clubbing has been reported in pulmonary alveolar microlithiasis, ${ }^{134}$ but not hypertrophic pulmonary osteoarthropathy. We report such a patient.

Department of Chest Diseases, Hacettepe University, School of Medicine, Ankara, Turkey

$S$ Emri

Emri

Z T Selplüuk

A A Sahin

Y I Baris

Reprint requests to: Professor Y I Baris

\section{Case report}

Pulmonary alveolar microlithiasis was diagnosed in a 44 year old woman and confirmed by open lung biopsy. Three years later she reported intermittent bilateral pain in the knees and ankles of four months' duration. The pain was associated with swelling at rest and aggravated by walking. She also noted increasing dyspnoea on exertion, and a cough producing yellow sputum. There was no relevant family history. On examination she was obese and had finger clubbing. Her wrists, ankles, metacarpophalangeal joints, and metatarsophalangeal joints were swollen, erythematous, and tender in response to light palpation. Auscultation of the chest disclosed bilateral basal end inspiratory crackles.

The erythrocyte sedimentation rate was $80 \mathrm{~mm}$ in one hour. Rheumatoid factor was present and $C$ reactive protein was increased. Arterial blood gases while she was breathing air were: $\mathrm{pH} 7 \cdot 42$, arterial carbon dioxide tension $\left(\mathrm{PaCO}_{2}\right) 4.2 \mathrm{kPa}$, arterial oxygen tension $\left(\mathrm{PaO}_{2}\right) \quad 9.9 \mathrm{kPa}$. Pulmonary function tests showed mild restriction and a transfer factor (TLCO) of $39 \%$ predicted. A chest radiograph (fig 1) showed very fine, sand like micronodules of calcific density, diffusely affecting both lungs. The appearance was unchanged from a radiograph taken 10 years earlier.

Computed tomography of the chest showed diffuse bilateral calcified densities, predomin-

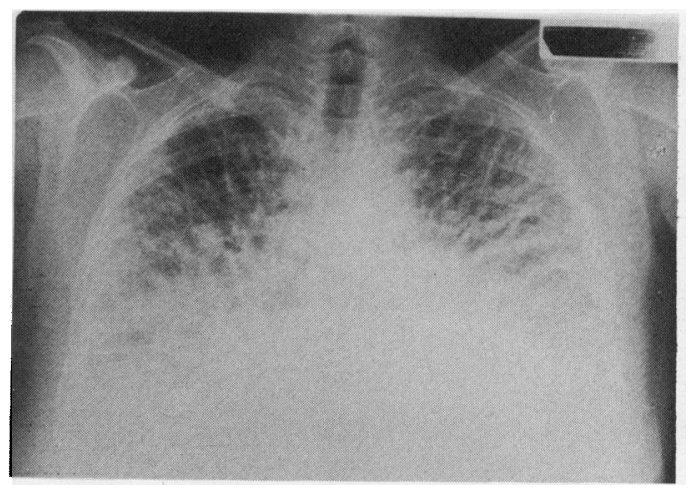

Figure 1 Chest radiograph showing very fine, sand like micronodules of calcific density in both lung fields. 